\title{
La Subjectivité du Bien-Être Dans la Santé
}

\author{
Célia Vaz-Cerniglia ${ }^{1,2, *}$, Céline Borg ${ }^{1,2}$ (D) \& Catherine Thomas-Antérion ${ }^{2}$ \\ ${ }^{1}$ Catholic University of Lyon, Lyon, France \\ ${ }^{2}$ University of Lyon 2, Lyon, France
}

\begin{abstract}
RÉSUMÉ - Dans cet article nous illustrerons l'accessibilité à un état subjectif à partir de deux situations cliniques. Nous observerons comment ces deux patients, l'un présentant accident cérébral vasculaire et l'autre, une pathologie neurodégénérative ont pu surpasser leur handicap et déployer leur potentiel créatif, passée la phase d'évaluation psychométrique.
\end{abstract}

MOTS CLÉS: bien-être, mieux-être, pathologie, évaluation, inter subjectivités, subjectivité

\section{The Subjectivity of Well-being in Health}

\begin{abstract}
In this paper we will illustrate the accessibility to a subjective state of well-being from two clinical situations. We will observe how these two patients, one suffered a stroke and one displaying a neurodegenerative disease, were able to overcome their handicap and deploy their creative potential, past the psychometric assessment phase.
\end{abstract}

KEYWORDS: well-being, pathology, evaluation, intersubjectivity, subjectivity

\section{A Subjetividade do Bem-Estar em Saúde}

\begin{abstract}
RESUMO - Neste artigo, ilustraremos o acesso a um estado subjetivo de bem-estar, a partir de dois casos clínicos. Observaremos como esses dois pacientes, um que sofreu um derrame e outro que apresentava uma doença neurodegenerativa, foram capazes de superar sua deficiência e implementar seu potencial criativo, passada a fase de avaliação psicométrica.
\end{abstract}

PALAVRAS-CHAVE: bem-estar, patologia, avaliação, intersubjetividade, subjetividade

La santé est définie comme un état ou un sentiment de bien-être. Plus précisément, "la santé est un état de complet bien-être physique, mental et social, et ne consiste pas seulement en une absence de maladie ou d'infirmité" (World Health Organization [WHO], 1946). Autrement dit, l'état de bien-être n'est pas seulement lié à l'absence d'une maladie et peut varier à différents niveaux d'avancée de la maladie. Maladie et santé ne sont pas des entités séparées et nous pouvons les considérer ensemble à différents degrés. Il s'agit d'un processus d'entropie qui est décodé par la manifestation des symptômes et des signes. Les symptômes correspondent à ce qu'énonce le malade. Les signes sont recueillis par le professionnel soignant, le médecin le plus souvent lors de l'entretien et de l'examen. Certains symptômes peuvent avoir valeur de signe (une céphalée unilatérale pulsatile au niveau d'une tempe est le signe d'une migraine). Les syndromes cliniques reposent sur l'association "symptômes et signes". En outre, la maladie revêt différentes significations selon la personne qui la considère : le malade, le médecin, le psychologue, la société. De plus, il est extrêmement réducteur de parler de "la" maladie. Celle-ci est multiple, de cause inconnue, connue et univoque (maladie infectieuse), mal connue ou plurifactorielle et pose quelquefois la difficile question

*E-mail: cvaz@univ-catholyon.fr

- Submetido: 26/10/2014; Aceito: 24/03/2017. 
du normal et pathologique. Le recueil des symptômes et l'analyse des signes permettent d'élaborer un diagnostic plus ou moins certain et avec une capacité ou non de guérison. Le fait d'aller mieux (de retrouver le bien-être) se distingue d'ailleurs du retour ad integrum. Le bien-être s'accompagne le plus souvent d'une adaptation à un nouvel état de santé et l'on voit bien que celui-ci peut s'observer y compris lorsqu'une maladie ou un problème social perdure. Le but du soin est de proposer tout autant une prise en charge médicamenteuse que non médicamenteuse.

L'objectif de cet article est de s'interroger sur cet équilibre homéostatique, cet état de bien-être, qui, outre le fait d'être malade ou non, est propre à chacun. Nous illustrerons ainsi nos propos en présentant deux situations cliniques: la première renvoie à une patiente qui suite à la survenue d'un accident vasculaire cérébral a dû dépasser cette crise et mobiliser de nouvelles ressources pour réajuster un équilibre de santé; la deuxième se centre sur un patient présentant une pathologie neurodégénérative. Ce type de pathologie entraîne une perte d'autonomie progressive, qui s'installe au fil des ans, et il semble difficile de maintenir un état de bien-être qui soit stable. Nous tenterons ainsi de réfléchir à un accompagnement possible dans cette situation d'invalidité progressive. Cette tentative d'ajustement ne s'établit pas seulement en fonction du soignant mais s'inscrit aussi dans une histoire personnelle, familiale, elle-même inscrite dans un contexte socio-politique et culturel. Il n'est pas neutre de rappeler que le sujet souffrant d'une maladie est souvent dénommé patient, suggérant par le signifiant même du mot combien l'emprise du soignant est grande et sachant que ce terme désignait dans le passé les condamnés à mort. Nous souhaitons notamment réfléchir aux rapports du sujet-soigné/soignant, à l'issue du diagnostic et de la prescription (l'ordonnance). Nous proposons comme situation de réflexion l'accompagnement du sujet dans une annonce diagnostique, avec d'une part, la nécessité de donner un sens entre la maladie objectivée (diagnostic) et d'autre part, le vécu subjectif de cette maladie (symptômes) avant la prise en soin. De plus, dans ce rapport intersubjectif, est pris en compte l'élaboration dans l'après-coup de ce qui se joue dans la relation tels que la dynamique transférocontre-transférentielle, l'identification, l'imaginaire etc. et il est important de souligner que la prise en soin est une forme de contrat et d'acceptation voire de choix tacites passés entre le soigné et le soignant. A tout moment ce contrat peut être résilié à la demande du soigné notamment qui montre par cet acte sa propre capacité à mener son existence pour atteindre un mieux-être. Nous verrons avec les deux situations présentées que ce contrat finalement peut se mutualiser en dépassant la situation duelle initiale.

En tant que psychologues, nous adoptons une approche transversale au niveau théorique et respectons les aspects pragmatique, écologique et non normatif du développement de l'être humain. Notre approche vise à respecter le sujet dans l'évolution de son désir et l'adaptation à sa nouvelle réalité (physique, mentale et/ou socio-familiale) à son rythme et avec ses moyens internes et externes. L'objectif de la prise en soin doit être défini avec le sujet. Dans certains cas, l'anosognosie/le déni du sujet peut être une limite. Il n'est pas plus éthique de suivre «à tout prix » le désir (irréalisable) d'un sujet que de lui imposer un réalisme pratique. En revanche, libérer un espace dédié à l'espoir s'avère primordial pour maintenir une prise en soin dynamique et respecter la part subjective du sujet. Il conviendra alors de cheminer vers cet objectif et l'objectivation de sa réalisation. Cette démarche est contenue comme nous le verrons par un cadre qui comporte des règles. Il existe néanmoins une part de liberté qui laisse place à l'émergence d'une créativité dans un environnement suffisamment étayant et empathique, pour inventer un soin, chaque fois singulier.

Comme énoncé précédemment, notre approche est transversale dans le sens où notre réflexion se nourrit de la psychologie clinique, développementale, expérimentale, physiologique et sociale. Ces dimensions sont amplement représentées dans le modèle d'Erikson (1950) qui rend compte de la manière dont tout sujet est confronté à des perturbations dans lesquelles se jouent des problématiques psychosociales impliquant la psyché, l'éthos et le soma. Ces tournants nécessaires pour se développer peuvent être structurants ou déstructurants sur le plan de la personnalité du sujet selon leur degré de résolution. Ainsi, la personnalité par le biais des stratégies mises en place par le sujet se transforme dans un sens comme dans un autre ou se maintient. On ne peut donc écarter aucune de ces orientations puisque des facteurs biologiques, psychologiques et sociaux et leurs interactions circulaires sont à l'origine de différentes pathologies physiques et mentales. Dans son modèle bio psycho-social, Engel (1980) avance que les aspects de la santé et de la maladie sont organisés selon un continuum du plus général au plus spécifique. A partir de ce modèle, on peut envisager le vécu d'une maladie comme la conséquence contextuelle, situationnelle d'un processus interactif entre d'une part, les caractéristiques mêmes d'une déficience et des incapacités de la personne, consécutives aux maladies et aux traumatismes et d'autre part, les caractéristiques mêmes de l'environnement qui peuvent être étayantes ou pas. En d'autres termes, il paraît évident qu'une personne qui se retrouve dans une situation invalidante, vit sa déficience, ses nouvelles incapacités selon ce qu'elle ressent et aussi selon ce qui fait sens d'après sa propre histoire, son inscription dans sa famille et dans la société. Il n'existe donc pas « une maladie » réduite à des symptômes connus qui font signe mais une maladie aux arborescences multiples selon la perception du sujet-soigné et la perception de son environnement. Ainsi, chaque maladie se colore différemment si nous privilégions la place du sujet, que son étiologie soit somatique et/ou psychologique.

La relation soignant/sujet-soigné bien que s'établissant dans un contexte écologique ne peut faire l'impasse de l'observation de la subjectivité du soigné. Le psychologue, soignant se met ainsi en lien avec un sujet-soigné et dans ce lien à l'autre, chacun doit retrouver son semblable. 
Le soignant est amené à respecter une certaine distance en vue de ne pas se laisser submerger par l'intensité des émotions transférées par le sujet-soigné et d'éprouver le mieux possible la relation instaurée. De plus, nous pouvons évoquer dans cette relation la notion de coping (Lazarus \& Folkman, 1984). Ce terme anglo-saxon renvoie à la notion de stratégie de faire face ou stratégie d'ajustement mise en place par l'individu et souligne le caractère évolutif et confrontatif des processus cognitifs et comportementaux déployés par celui-ci. Ce terme ne doit pas être confondu avec la mise en place de mécanismes de défense. En effet, le soigné dans un souci d'adaptation met en place des stratégies face à un changement, une crise, qui peuvent être très importants, voire dépasser ses anciennes ressources adaptatives. Selon le modèle de Lazarus et Folkman, il s'agit ainsi d'amener le sujet (en respectant son rythme et en limitant les abréactions) à reconnaître le changement, à identifier une crise, d'en évaluer l'intensité, d'examiner des réponses d'adaptation potentielles, en vue de la dépasser plus sereinement. Pour que ce processus transactionnel prenne sens, pour que de nouvelles réponses adaptatives émergent, il peut-être envisagé une éducation thérapeutique. Il s'agit d'une prise en soin continue qui s'adresse aux personnes en souffrance afin de leur permettre une meilleure acceptation d'eux-mêmes. Ce processus de soin amène les professionnels à transmettre leur savoir-faire, leur savoirvivre en redéfinissent les besoins, les priorités de vie des sujets-soignés. S'appuyant sur cette interactivité, nous pouvons considérer que la prise en soin est modulée en fonction de la personnalité, des connaissances du patient ainsi que de celles du soignant. Dans ce processus, il est essentiel de considérer l'origine du conflit, le moment de la crise ou de la maladie (et son pronostic), la personnalité du patient et les ressources dont il dispose pour s'adapter, ainsi que le contexte socio-familial de celui-ci. Le psychologue est un soignant qui recherche une alliance thérapeutique pour garantir un contrat entre un sujet-soignant et un sujet-soigné. Il est ainsi essentiel de souligner dans l'articulation du soin la volonté de comprendre cette dynamique qui mène du soigné-sujet au sujet-soigné en vue de garantir une distance sans cesse ajustée en combinant les notions d'objectivité et de subjectivité comme précédemment énoncé.

Nous faisons l'hypothèse que pour atteindre un mieuxêtre, le sujet soigné nous amène à reconsidérer sans cesse sa singularité. Pour répondre à cette hypothèse, nous proposons de modéliser la relation thérapeutique qui peut s'installer entre le soignant et le soigné à partir de deux cas cliniques. L'intérêt de modéliser ces situations cliniques nous permet de ne pas perdre le fil conducteur, de mettre en scène l'histoire d'une rencontre ponctuée par une succession d'épisodes pro grédients, ré grédients, stagnants, voire dégénératifs. La première situation concerne la rencontre de $\mathrm{MB}$, une jeune femme trentenaire qui, suite à un accident vasculaire cérébral, a ressenti une modification subjective émotionnelle et des douleurs neuropathiques hémicorporelles droites très invalidantes. La deuxième présente l'accompagnement de
Monsieur AC souffrant d'une démence neurodégénérative. La première rencontre évoque une relation qui sous-entend de récupérer et/ou de compenser ses difficultés pour dépasser la crise. La deuxième évoque une relation impliquant une perte d'autonomie et d'indépendance progressives et questionnant l'accompagnement thérapeutique. Dans les deux contextes, il s'agit d'une observation longitudinale soucieuse du rythme et du désir du sujet. Il s'agit aussi d'une prise en compte de la détresse du sujet-soigné, de l'aidant et du soignant professionnel. L'accès au soin se met en place selon la représentation du sujet qui peut l'enfermer dans une relation binaire. Par exemple, le fait de consulter un neurologue et un neuropsychologue pour une rééducation de troubles mnésiques et d'occulter d'éventuels troubles psychiques ou encore de consulter un psychiatre pour bénéficier d'un traitement antidépresseur sans reconnaître d'éventuels troubles neurologiques.

Nous constatons que suite à une volonté première d'objectivation pour porter un diagnostic précis et répondre à la première demande, celle d'être soigné, nous nous acheminons vers la reconnaissance du sujet dans sa singularité non réduite à sa maladie. En effet, dans un premier temps s'initie un travail d'investigation de la symptomatologie, du retentissement relatif de la crise sur le quotidien et l'autonomie du sujet; dans un second temps, les préoccupations du soignant se centrent sur le désir du sujet, ce dernier pouvant s'inscrire dans une demande modifiée par le sujet lui-même au cours de nos rencontres intersubjectives qui laissent de moins en moins de place aux symptômes (et donc à la maladie)

Dans un contexte de modélisation écologique, le schéma (figure 1) qui dispose d'un axe diachronique montre qu'à l'instant $\mathrm{T}(\mathrm{n}=1)$, une personne lambda se situe dans un état ordinaire, n'étant confrontée à aucune situation psychosociale conflictuelle particulière. A un instant $\mathrm{T}+1$, consécutif à un changement radical, cet état initial commence à dévier de l'état ordinaire. Le sujet devient alors malade car son comportement ne correspond plus à ce qui est attendu socialement et selon ses références. Cet écart peut aboutir à un conflit intra psychique conséquent, largement dépendant des capacités adaptatives du sujet. Dans un premier temps, il consulte pour guérir à tout prix de sa maladie et retrouver le cours ordinaire de sa vie (temps $n+1$ prégnance de la maladie). A la phase suivante, il retrouve son statut plein de sujet-soigné "sain ou guéri" au détriment de celui de malade. Ce changement ne peut s'effectuer que s'il s'octroie (part active) et que s'il bénéficie (part de l'environnement) d'un espace transitionnel propice à l'expression de son désir et de sa créativité. Le conflit intra psychique devient moins intense. Les deux situations suivantes nous permettent de suivre ce processus de perte puis de réappropriation de l'existence à travers celle de sa subjectivité inscrite dans un cadre familial notamment, cadre qui contient le sujet et maintient le fil de son histoire au moment d'une défaillance psycho-corporelle (cf. figure 1., $\mathrm{n}+1+1=1$ ). 


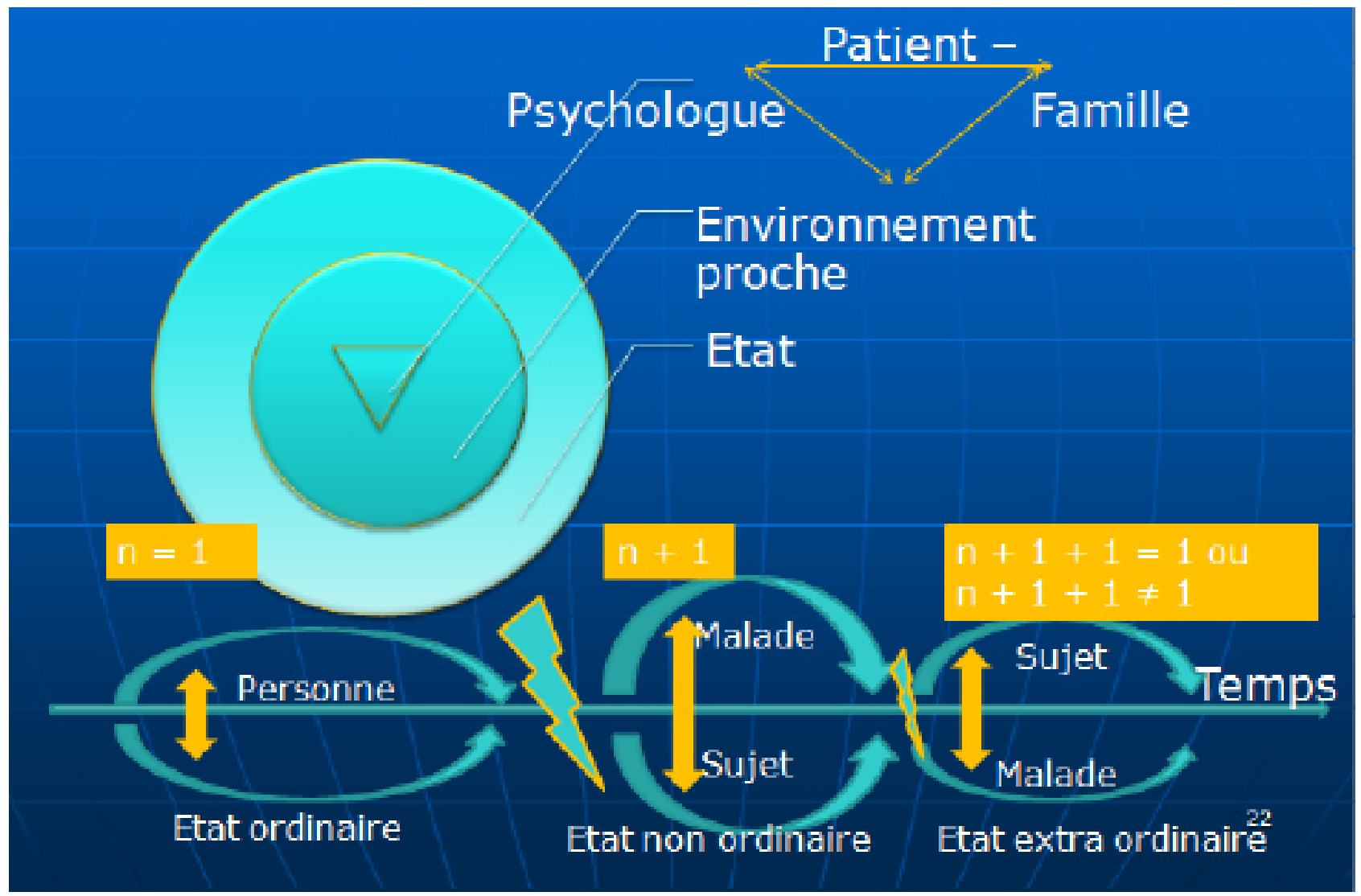

Figure 1. Contexte écologique (Vaz-Cerniglia, 2006)

\section{SITUATION DE MADAME MB}

Nous allons discuter la situation de MB, une jeune femme de 36 ans, ancienne coiffeuse de métier et qui est venue en consultation en février 2008 au CAJ (Centre de rééducation neuropsychologique et d'accompagnement social) de L'Adapt-Rhône. Celle-ci a présenté un AVC localisé dans la région sylvienne gauche en février 2007. Une IRM pratiquée en février 2008 a objectivé une lacune au niveau de l'insula postérieure et du cortex somatosensoriel secondaire (SII) à gauche. La patiente a présenté initialement une hémiparésie droite et une aphasie de conduction, mais a très rapidement récupéré de ces symptômes. Au moment de la consultation, l'examen neurologique objective un léger déficit de la main droite et une hémianesthésie hémicorporelle droite. Il existait aussi des signes d'allodynie, avec des sensations de brûlures permanentes majorées notamment par le contact cutané et par le froid. Du fait du handicap physique (léger), MB ne peut plus exercer son travail de coiffeur et suit un parcours de réinsertion avant une orientation en UEROS (Unité d'Evaluation, de Réentraînement et d'Orientation Sociale et professionnelle). Elle rencontre au début de ce parcours dans le cadre de l'élaboration du Projet de vie, le médecin. MB est mariée et vit en couple avec un fils issu d'une première union. Originaire de l'île Maurice, elle vit loin de sa famille mais entretient avec elle des liens réguliers et des communications via internet. Elle est très entourée par un réseau amical solide et sa belle-famille. La patiente est anxieuse quant à son avenir mais de façon adaptée, elle ne présente pas d'élément dépressif majeur. Interrogée sur le caractère douloureux de l'hémianesthésie, la douleur s'avère être très sévère ( $9 / 10$ sur EVA) et répondre aux caractères d'une douleur neurologique centrale. MB à l'évidence si elle s'est adaptée à l'anesthésie hémicorporelle voire a accepté sa réinsertion professionnelle, présente un état de mal-être somatique préoccupant. La douleur est telle qu'elle restreint son autonomie et induit des comportements de stress majeur. MB ne va plus au supermarché, ne supportant pas le froid des rayons réfrigérés, dort avec des gants chauds ou va déjeuner chez des amis ou au restaurant avec ses couverts qu'elle choisit en plastique pour ne pas risquer de toucher du métal froid. Cette plainte a été considérée jusque là comme amplifiée ou de rajout. La relecture de l'imagerie cérébrale confirme que la lésion concerne l'insula, région du cerveau dont l'atteinte entraîne des tableaux douloureux majeurs. MB est alors soumise à des mesures 
électrophysiologiques qui confirment la symptomatologie douloureuse et notamment sa gravité. MB est informée du diagnostic de douleur centrale post AVC. Elle témoignera de « son soulagement psychique » de cette annonce, ayant pensé parfois et ce notamment devant l'incompréhension des médecins de sa situation, être folle. Des traitements antalgiques sont alors prescrits avec beaucoup d'aléas. MB est calmée à $75 \%$ par l'association de 3 produits pris à fortes doses (qu'elle équilibrera elle-même). MB est d'emblée très active et participative dans l'équilibre du traitement. MB estimera en 2009 ne pas avoir récupéré son intégrité mais avoir considérablement amélioré sa vie et évaluera alors sa douleur à 4/10 (tolérable) et son bien-être physique à 8/10 . Néanmoins, elle sera amenée à accepter les effets secondaires des traitements (prise de $20 \mathrm{kgs}$ en une année). Elle décrira alors un nouvel état de mal-être somatique et psychique : "je suis grosse et vilaine, moi qui avait la taille de guêpe. J'ai du changer tous mes vêtements". Elle entamera des régimes sans succès. Elle consultera de nombreux thérapeutes et elle demandera à se faire poser un anneau digestif en 2010. Elle considérera cette chirurgie comme un succès malgré la mutilation et le changement total alimentaire et considérera sa vie comme transformée. Jamais, elle n'envisagera d'arrêter les traitements antalgiques.

Sur le plan cognitif, un bilan orthophonique et neuropsychologique est réalisé au moment de son admission et n'a pas montré de modifications cognitives, si ce n'est quelques légères difficultés exécutives et de la mémoire de travail. Compte-tenu du bilan entrepris, plusieurs entretiens avec $\mathrm{MB}$ et le neurologue sont survenus. Il faut souligner que MB très intéressée par toutes les explications scientifiques fournies et a adopté une posture classique chez les sujets porteurs de maladies (douleurs) chroniques dont la situation clinique (lésion insulaire) est très rare, se montrant très participante pour la réalisation de bilans. Les thérapeutes doivent bien sûr faire la part des choses entre un sujet qui se donne en pâture aux médecins qui l'ont guérie et un sujet souhaitant d'une part avoir une information fine sur ce qui lui arrive et d'autre part étant prêt à participer à des expériences utiles aux connaissances (et donc à d'autres sujets).

Sur le plan comportemental, MB exprime lors d'un troisième entretien neurologique un changement émotionnel. Elle parvient parfaitement à décrire les situations émotionnelles et sociales et à interagir avec autrui (au CAJ, elle se montre empressée auprès de sujets très handicapés et décode très vite les petits conflits et les frustrations des uns et des autres lors des activités de groupe) mais elle ressent un émoussement du ressenti de ses propres émotions. Par exemple, avant son AVC, MB avait peur de prendre l'avion, tandis qu' après la survenue de son AVC, elle ne ressentait plus cette crainte. Ou encore, suite à son AVC, elle était partie rejoindre sa famille à l'île Maurice, et à son arrivée, elle fut surprise de ne pas ressentir une joie intense à l'accueil de sa famille, alors qu'elle décodait complètement la joie de celle-ci à la recevoir ou encore, elle ne ressentit pas la peine habituelle de laisser son fils en France et ne pleura pas comme à l'accoutumée pendant le voyage. Elle évoqua plus qu'un mal-être (avec les repères qu'elle en a concernant l'état somatique), un sentiment d'étrangeté et d'inconfort. Le neurologue confirma cette modification à l'aide de l'échelle des changements de personnalité d'IOWA. Cette échelle est remplie par un proche de l'entourage et comporte 29 dimensions évaluant ainsi différents aspects du comportement et de la personnalité. La variable dépendante analysée ici est le score obtenu dans chacun des 29 domaines de la personnalité grâce à une échelle de points allant de 1 à 7 , permettant d'avoir ainsi une vision de l'estimation de la conscience du changement pour le patient. Les résultats ont montré un changement significatif du comportement avant (avec un score à 97) et après l'AVC (avec un score à 50) notamment pour les items signifiant des changements émotionnels: «irritabilité», «sensibilité», «émotion inadéquates», «se sent débordée». MB verbalisa alors sa satisfaction que l'on puisse «mesurer sa gêne ».

Elle accepta alors une évaluation neuropsychologique plus fouillée, marquant comme pour l'étude de la douleur, une vraie curiosité. Après un entretien, la neuropsychologue a réalisé une tâche informatisée et a montré à l'aide d'une tâche d'identification des expressions faciales et d'évaluation de l'intensité de ces expressions, que la patiente identifiait correctement celles-ci. En d'autres termes, elle parvenait à identifier (comme elle le disait) les émotions de joie, de peur, de dégoût... En revanche, l'expression émotionnelle des visages et ce quelle que soit l'émotion (positive ou négative) était traitée comme moins intense, comparativement aux sujets contrôle. Il a été considéré que cette symptomatologie pouvait être associée à la lésion cérébrale, localisée dans le sous-cortex, modifiant des mécanismes impliqués dans un état interne non conscient de ressenti des émotions des autres. MB n'améliora pas cet état mais fit part de l'aide que l'explication organique de ces symptômes lui apportait.

Une particularité clinique exceptionnelle de cette situation est que MB s'est mise à peindre six mois après son AVC et six mois avant d'intégrer le CAJ. Nous rappelons que cette patiente a un CAP de coiffure et n'avait jamais auparavant ni dessiné ni peint et n'avait pas d'intérêt pour l'art. Six mois après son AVC, elle a ressenti le besoin de peindre, est allée acheter des toiles, des pinceaux et de la peinture à l'huile et a produit son premier tableau

Par la suite, elle a continué ainsi à peindre presque quotidiennement et continue encore. Elle peut interrompre son activité, mais néanmoins elle ressent un besoin irrépressible de se mettre à peindre et peut décaler pour cela des activités ménagères urgentes (préparation des repas). L'apparition de production artistique suite à une lésion vasculaire est exceptionnelle, le nombre de cas dans la littérature publiés après un AVC se compte sur les doigts des deux mains. La patiente apporta à la neurologue, «son book». Il s'en suivit une étude spécifique (avec l'accord de MB) de sa production. Celle-ci était réalisée très majoritairement avec des couleurs chaudes. 


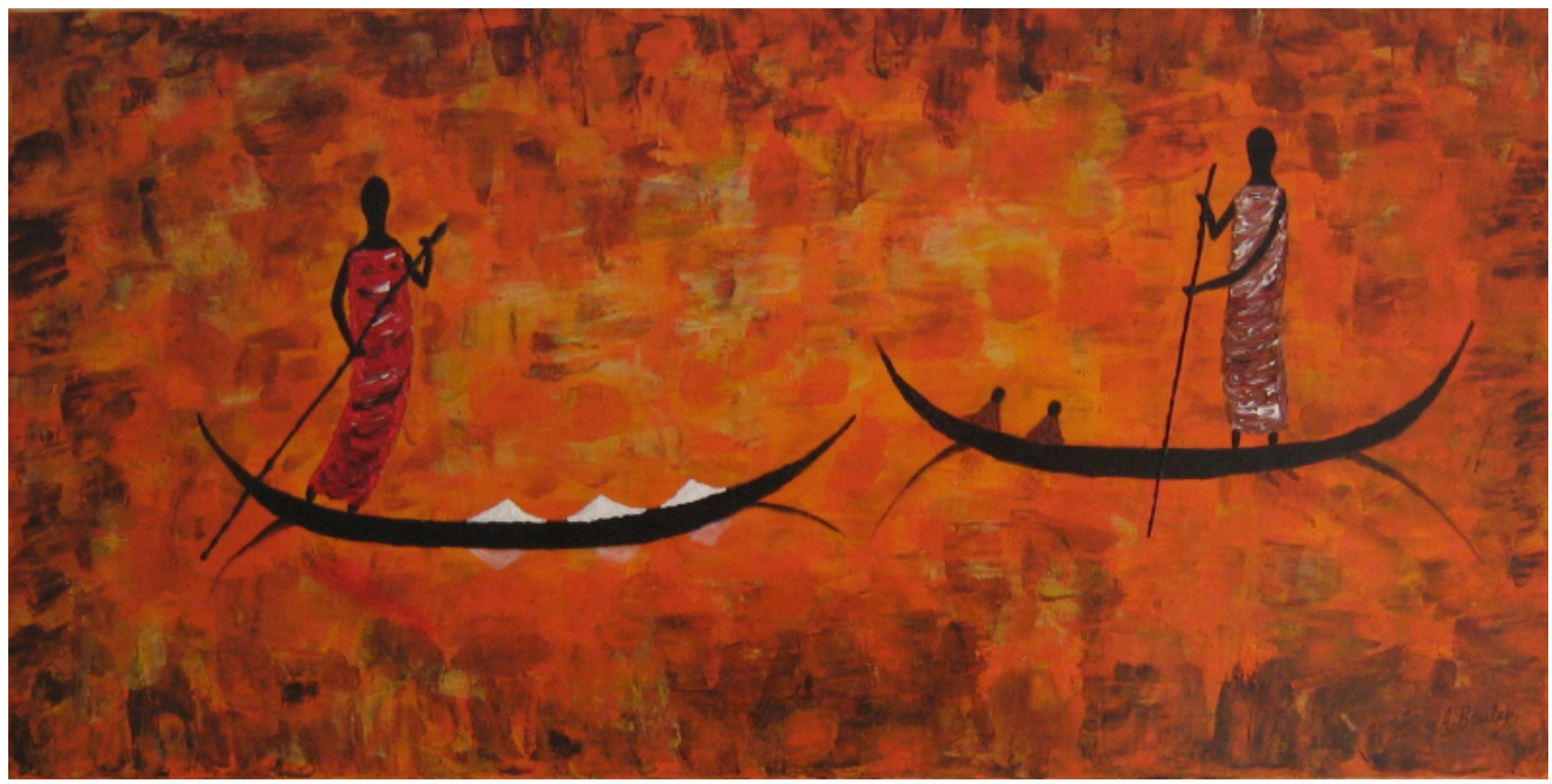

Figure 2. Peinture MB. Bateaux

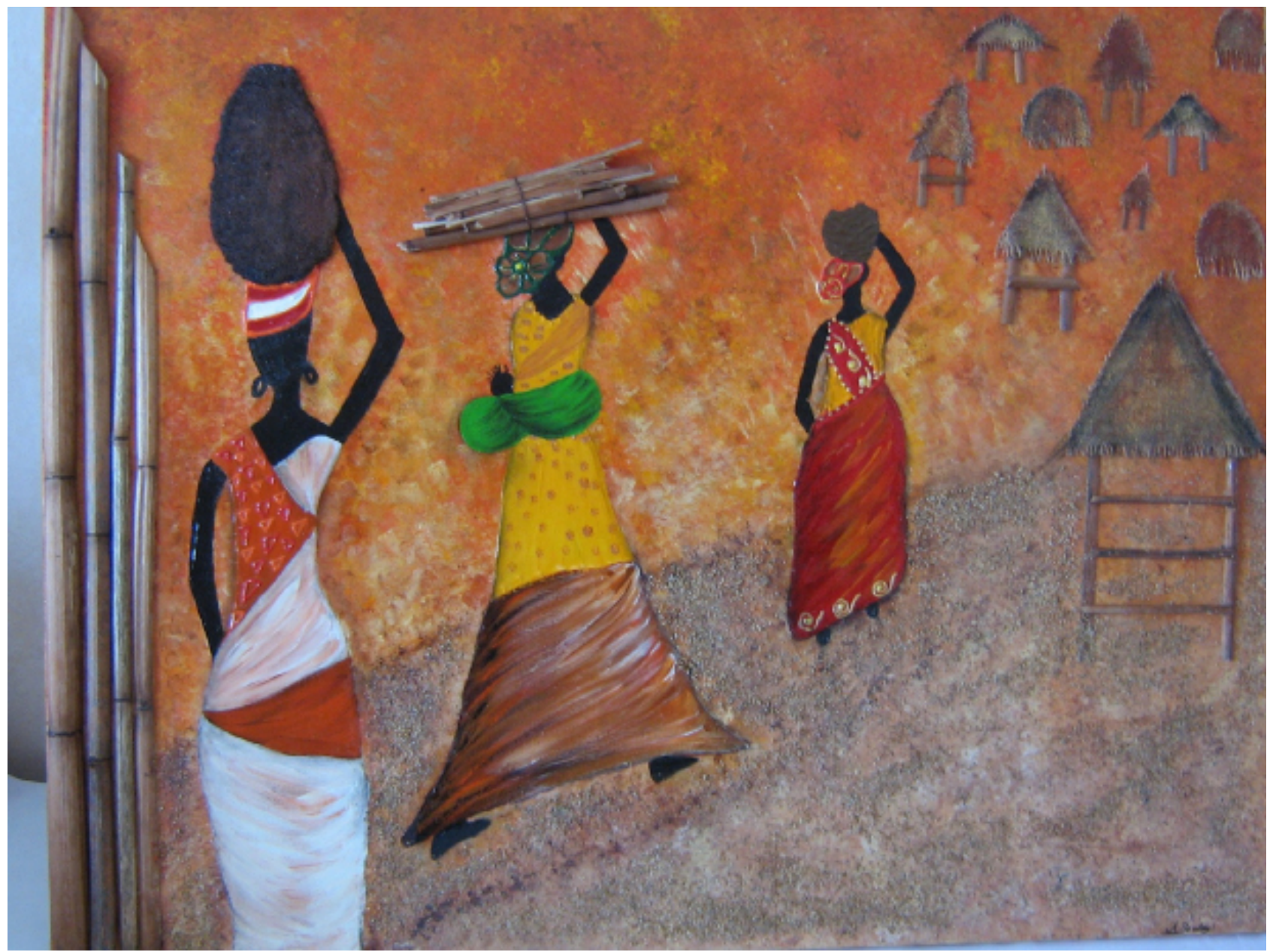

Figure 3. Peinture MB. Village 
MB évoqua le fait que les couleurs chaudes diminuaient le seuil de ses douleurs et que les couleurs froides (qu'elle évitait d'utiliser) les augmentaient ! L'hypothèse de synesthésies douleur-somesthésie-couleur-chaleur fut suggérée. La qualité artistique de ses peintures donné lieu à une publication scientifique (nous ne développons pas cet aspect pas plus que le fait que MB accepta de participer aux travaux d'un spécialiste travaillant sur la couleur qui donnèrent également lieu à une publication dans une revue internationale). MB développa elle-même une activité antalgique et améliora de façon conséquente son bien-être physique. Elle la poursuivit après l'amélioration médicamenteuse avec le même plaisir. Cette activité très envahissante conduisit $\mathrm{MB}$ à participer à des salons de peinture amateurs et elle en tira un bien-être psychologique et socio-familial évident. Enfin, MB suivit un parcours de réinsertion professionnelle et intégra en 2011 une activité à mi-temps de manucurie avec succès. Elle retrouva ainsi un travail en milieu ordinaire, retrouvant une place sociale satisfaisante.

Cette situation clinique exceptionnelle souligne la restauration du bien-être, sans la disparition du déficit neurologique. Elle distingue mal-être et inconfort. Elle montre l'intrication du mal-être somatique et psychique (et social même si la patiente supportait assez bien sa perte d'emploi). Elle rappelle que leur amélioration ne va pas forcément de pair. La restauration du bien-être démarre ici dès le bilan neurologique avec l'écoute des symptômes de MB (douleur au froid), l'examen des signes (hémianesthésie), l'évocation d'un syndrome neurologique (douleur centrale) et bien sûr l'annonce d'un diagnostic. La même remarque peut être faite pour le bilan neuropsychologique très spécialisé : symptômes (émoussement émotionnel), signes (expression faciale), syndrome (modification analyse automatique de l'état interne) et l'explication de celui-ci. La prescription de traitements participe à la restauration d'un état de santé satisfaisant: prescription d'antalgiques et traitement chirurgical de leurs effets secondaires (anneau gastrique). L'accompagnement du projet professionnel (que nous ne développons pas) évidemment apporte une aide concrète et psychologique à la patiente ! "L'intérêt scientifique» des chercheurs et la participation active de MB sont des éléments importants de ce parcours. Enfin, l'expression artistique restaure MB dans les dimensions somatique, psychique et socio-familiale. Cette observation démontre qu'après une maladie neurologique grave (AVC), invalidante socialement (perte d'emploi), des processus positifs peuvent être observés (expression artistique) montrant bien que le bien-être peut accompagner une situation pathologique et permettre à une personne malade de retrouver un «état de fonctionnement somatique, psychique et social normal».

\section{SITUATION DE MONSIEUR AC}

Monsieur AC, présentait une démence précoce qui n'était pas encore diagnostiquée à l'âge de 56 ans et à la demande de son médecin, il est venu consulter une psychologue au sein de son cabinet libéral pour une évaluation de ses fonctions cognitives. Lors de son premier entretien, ce patient n'a pu sortir du cabinet du psychologue, perdu dans les locaux. La psychologue a appris que Monsieur AC, accompagné par sa compagne continuait à conduire. Ce patient était en arrêt maladie sans vraiment comprendre la raison, si ce n'est qu'il rencontrait de plus en plus de difficultés à assumer son travail qui exigeait de nombreux déplacements en voiture ainsi qu'une supervision de travaux dans le bâtiment.

Deux évaluations cognitives ont été réalisées pour suivre l'évolution de son état. La première a eu lieu en décembre 2004 et immédiatement après, Monsieur AC a été pris en charge par la psychologue, à raison d'une fois toutes les deux semaines. En parallèle, il était suivi également par un neurologue qui a, après plusieurs bilans neurologiques, communiqué sur le type de démence dont il était atteint, annonce qui a permis de confirmer ce que lui et les siens pressentaient, mettant un terme à une recherche effrénée du pourquoi et du pour quoi. Au cabinet de psychologie, le travail a surtout été axé dans un premier temps sur la rééducation (statut soigné-sujet avec les symptômes en premier plan) et progressivement s'est orienté à la demande du patient vers un travail sur le tournant de son existence, sur sa capacité à être seul et avec les autres (statut de sujet-soigné avec la subjectivité en premier plan). L'aspect affectif s'avérait primordial afin d'accepter cette maladie qui progressivement est apparue comme inéluctable, accompagnée de changements irrémédiables dans sa vie et dans celle de son entourage proche. D'une évaluation à l'autre (2004 à 2007), les performances de monsieur AC diminuaient progressivement mais certaines de ses facultés restaient satisfaisantes. Par exemple, pendant longtemps, il a réussi à former correctement des concepts au test des similitudes de la WAIS III (Wechsler, 1997). Sa mémoire était déficitaire mais il réussissait à retrouver des éléments par l'indiçage. En revanche, d'autres traitements comme les traitements perceptifs, visuo-spatiaux, nécessitant un coût attentionnel, ou encore les praxies étaient déficitaires. Par exemple, dans le test de complètement d'images de la WAIS III, où l'on présente une série de dessins sur lesquels il manque systématiquement un détail, qu'il faut retrouver, monsieur $\mathrm{AC}$, très rapidement ne parvenait pas à appréhender l'élément pertinent absent de la scène visuelle. Cette incapacité pouvait expliquer sa difficulté au quotidien d'explorer son environnement. Néanmoins, comme ses facultés de raisonnement et de compréhension se sont maintenues pendant un certain temps, une alliance thérapeutique a pu se mettre en place favorisant un travail d'introspection avec la psychologue. Ce sujet-soigné s'est 
saisi de cet espace-temps singulier en exploitant tant bien que mal le canal langagier. En dépit de son trouble du manque du mot, il s'est autorisé à s'exprimer de plus en plus sur l'époque pas si lointaine où il était actif professionnellement, sur sa contribution au sein de sa famille, allant même jusqu'à révéler des informations jusque là refoulées. Ces souvenirs convoqués explicitement pour la première fois en présence d'une personne nous apparaissent ordinaires dans le cadre d'un accompagnement thérapeutique, que le sujet soit jeune ou plus âgé. Cependant, ce patient atteint de démence, voyant son état de bien-être irrévocablement se dégrader a pu vivre un autre état, propice au retour de faits chargés d'émotions, sans éprouver aucune gêne. Cette observation peut se rapprocher de l'état de transparence psychique de la femme enceinte (Bydlowski, 2000). Le fait que ce sujet abandonne tout désir de guérison, souhait initialement tant attendu par lui et son entourage, a été rendu supportable par le vécu de cet état extraordinaire transcendé par une tonalité émotionnelle. Le fait aussi de pouvoir dans un cadre d'alliance thérapeutique aisément livrer à son sujet-soignant des informations inavouables peut être interprété comme un espace libéré des contraintes sociétales, familiales et morales. Partager ces informations authentiques et personnelles a contribué à un état de mieux être, autrement dit, a permis de renouer avec son narcissisme.

Ce processus thérapeutique étayant a recentré le sujetsoigné dans sa propre capacité à désirer et à décider. Monsieur $\mathrm{AC}$ a commencé à prendre des initiatives personnelles dans l'espace réduit qui lui était octroyé (environnement familial très protecteur) et ceci malgré un défaut de participation plus active dans les tâches quotidiennes notamment, comme dresser la table en prenant soi de mettre les couverts. Ce déclin désespérait particulièrement son entourage qui voyait là autant d'exercices rééducatifs capitaux gâchés pour eux. La répétition de tâches quotidiennes maintenait une capacité à raisonner sur le plan perceptif, proprioceptif et visuospatial, ralentissant le processus de dégénérescence en attendant le médicament miracle. Ce sujet-soigné avait saisi avec davantage d'acuité d'autres objets d'investissements propices à l'amélioration de son état alors que ses proches s'arrimaient encore aux pouvoirs illusoires de guérison de la rééducation selon lui. Puis comme dit précédemment, l'objectif des entretiens psychologiques ne s'est plus centré sur la rééducation qui répondait au départ à un désir de retrouver ses facultés cognitives et de retrouver sa position d'avant (place dans la société; cf. figure 1: $\mathrm{n}=1$ ) mais à une demande d'être écouté dans la réminiscence de ses vécus sur les plans socio-affectif et socio-professionnel. Il avait aussi besoin d'être contenu dans sa souffrance car pris dans des accès d'angoisse où il se sentait tomber sans pouvoir trouver une accroche pour limiter sa chute. Ainsi, en lieu et place de la mise en application de certains exercices au quotidien, un espace s'est enfin instauré au sein des rencontres sujetsoigné/soignant propices à l'expression de soi (cf. figure 1: $\mathrm{n}+1+1=1$ ). Monsieur $\mathrm{AC}$ a commencé à évoquer les figures auxquelles il s'identifiait et qui lui étaient chères, telles son grand-père qui était un homme robuste, de grande stature à l'instar du Général de Gaule et qui en imposait (essayait-il de dire) au moyen d'un lexique de plus en plus restreint du fait de l'évolution de la maladie. Ces références issues de sa mémoire autobiographique qui correspondent à un certain idéal du moi sont venues consolider l'auto-estime du sujetsoigné. En effet, ce sujet avait hérité de ces qualités et avait réussi dans sa vie professionnelle. Plus tard, à la naissance de son petit-fils il a confié à sa psychologue qu'il lui avait transmis sa stature et peut-être encore davantage, qu'il serait entrepreneur comme lui-même l'avait été et que ce petit fils n'avait pas hérité de son fils, petit comme sa femme, insuffisamment intrépide. Il imaginait ce petit-fils grandir et le soignant l'a accompagné dans cette rêverie où tout était envisageable, possible. La communication s'est poursuivie avec les membres de sa famille et le médecin de famille. En l'occurrence, la psychologue a accordé une écoute attentive et bienveillante envers la compagne extrêmement préoccupée par l'état de son mari au point de songer à se supprimer à la mort de celui-ci. Elle a accordé un temps d'écoute au fils qui recherchait des conseils avisés pour apprendre petit à petit à comprendre et tempérer les accès de colère et d'impatience de sa mère pour éviter d'enfermer, de réduire son père à l'état de malade. Son fils a saisi l'importance de partager des moments avec son père alors qu'il allait devenir père à son tour. Ces moments pouvaient revêtir des activités telles que se rendre à la piscine car la marche devenait de plus en plus problématique, en prenant soin de veiller sur son père car dans l'incapacité de ranger ses affaires, de s'habiller, de s'orienter au sein du bâtiment municipal. Cette communication s'est orienté vers plusieurs axes, celui entre les différents professionnels partageant un point de vue convergent (cf. figure $1: n+1+1=1$ ) et offrant un cadre étayant semblable, celui entre les membres de la famille actuels qui recherchaient un maillage soutenant et entre les ascendants et descendants s'exprimant dans une transmission intergénérationnelle. Laisser sa place prenait de plus en plus sens chez ce sujet-soigné qui allait perdre bientôt et de façon irrémédiable tout désir, de vie ou/et de mort. En effet, quelques mois plus tard sa femme a appelé la psychologue pour lui annoncer la mort de Monsieur AC. Elle continue à être suivie par le médecin de famille et s'occupe souvent de son petit-fils, fortement identifié à la figure de son mari. 


\section{CONCLUSION}

Ces deux situations montrent que le professionnel soignant et considéré comme expert dans son domaine apprend toujours de son semblable, du sujet-soigné. Cette reconnaissance favorise un espace de créativité indispensable pour continuer à se projeter, à transmettre, à apprendre. En effet, cet espace partagé se crée d'abord dans un rapport duel et fait émerger d'autres dimensions que celles purement normées et évaluatives sur le plan quantitatif. Ce rapport se modifie pour privilégier un contrat mutuel entre les différents sujets comme nous le découvrons dans les deux situations présentées avec Madame MB et Monsieur AC. Nous nous rendons compte qu'en tant que soignant, qu'enfant, que conjoint nous continuons à apprendre du sujet-soigné tout autant que nous apprenons sur nous-mêmes dans nos représentations, nos projections et dans notre pratique en l'enrichissant. Avec Madame MB, nous découvrons et apprenons que le sentiment de bien-être peut se vivre pleinement en dépit d'une maladie présente qu'elle a réussi à mettre au second plan. Avec Monsieur AC, un accompagnement thérapeutique intégrant les membres de la famille proche a permis d'accéder à un espace de liberté d'expression pour chacun. Chaque membre a pu cheminer à son propre rythme pour accepter la mort. Nous constatons après coup que le sentiment de mieux-être ou encore d'intégrité selon Erikson (Erikson, 1950) a pu être atteint par le sujet à partir du moment où il a été convaincu de l'utilité de son existence en s'inscrivant lui et les siens dans une transmission à la fois filiative et affiliative. Auteur de cette démarche et reconnu par les siens et le soignant, il a pu plus aisément atténuer sa souffrance et évoluer en dehors de la norme sociétale au profit d'un développement psychoaffectif, cognitif et social plus respectueux de sa situation et de sa personne.

En conclusion, nous pouvons dire que par l'intermédiaire de ces sujets qui viennent à notre rencontre et nous inspirant de la pensée de Bion, nous nous appuyons sur l'appareil à penser de nos patients pour enrichir notre propre capacité à repenser, pour revisiter nos croyances et pratiques. Nous ne nous limitons pas à prêter notre appareil psychique, en cela nous soulignons l'aspect mutuel du contrat passé avec le sujetsoigné. Chaque situation pathologique est ainsi appréhendée dans sa singularité en mettant en premier plan le sujet.

\section{REFERENCES}

Bydlowski, M. (2000). Je rêve un enfant. L'expérience intérieure de la maternité. Paris: Odile Jacob.

Engel, G. L. (1980). The clinical application of the biopsychosocial model. American Journal of Psychiatry, 137, 535-544.

Erikson, E. H. (1950). Enfance et société. Neuchâtel: Delachaux et Niestlé.

Lazarus, R.S., \& Folkman, S. (1984). Stress, appraisal and coping. New York: Springer.
Vaz-Cerniglia, C. (2006; 26-27 nov.). À 57 ans, comment vivre avec une incapacité nouvelle? Troubles de la coordination visuomotrice, visuoattentionnelle et visuospatiale. Poster session presented at Perspectives psychologique et neurologique $-6^{\text {ème }}$ congrès ARIBa. Lyon: Centre de Congrès.

Wechsler, D. (1997). Wechsler adult intelligence scale-Third edition. San Antonio: The Psychological Corporation. 